\title{
Microstructural characterization of copper films produced by electrodeposition
}

\author{
R. F. Santos* , F. Viana* \\ * CEMUC, Department of Metallurgy and Materials Engineering, University of Porto, R. Dr. Roberto Frias, \\ 4200-465 Porto, Portugal
}

Email: ruben.santos@fe.up.pt

To supply the electronic devices market's constant demand for more, smaller and more powerful chips, interconnects are made thinner and thinner increasing the current density, the overall chip heating by power dissipation and the proneness to circuit disruption by mechanical and electromigration failure. In order to improve the performance of copper interconnects it has been proposed the use of pulsed current (PC) electrodeposition instead of direct current (DC) used by the state of the art dual damascene process. Studies have reported for pulse electrodeposited copper films good mechanical strength combined with higher electrical conductivity and higher thermal stability than DC films, due to the presence of nanoscaled twins [1-4].

The objective of this work was to characterize electrodeposited copper films produced by both direct and pulsed current modes. Copper films with thicknesses ranging between 30 and $50 \mu \mathrm{m}$ were deposited onto a graphite polished substrate in an additive-free copper sulfate electrolyte. The films were detached and fine polished on the film-substrate interface for electron backscatter diffraction (EBSD) analysis; 3 millimeter diameter discs were cut off from the films, grinded and jet polished for transmission electron microscopy observation (TEM).

Orientation maps are depicted on figure 1 for DC and PC films in which grain boundaries, outlined in black, were set to exist above a $15^{\circ}$ misorientation between domains. DC shows a finer microstructure than the PC mode, with an average grain size of 221 and $433 \mathrm{~nm}$, respectively. The primary and secondary twin boundaries along the $\{111\}$ and $\{110\}$ planes are outlined in red. The histogram depicted on figure 2 displays a grain size distribution determined by EBSD where it is observed that the majority of the grains are $200 \mathrm{~nm}$ or less for DC while for PC smaller grains coexist among grains as large as $600 \mathrm{~nm}$ and above which contain many nanoscaled twins. The average grain size was determined using the grains area, assuming each grain as an equilateral hexagon and the grain diameter the length between two parallel sides. DC and PC modes presented a twin-to-grain boundary ratio of 0.30 and 0.62 , respectively. As the resolution for EBSD analysis is fixed around $50 \mathrm{~nm}$, the smaller grains and twins could not be observed by this technique. However, TEM images shown on figure 3 reveal the presence of numerous nanoscaled twins for PC while DC films present a near twin-free microstructure.

To "Centro de Materiais da Universidade do Porto" in the person of Rui Rocha. This work was supported by "Fundação para a Ciência e a Tecnologia" (FCT) through the grant SFRH/BD/61827/2009 and sponsored by FEDER funds through the program COMPETE - Programa Operacional Factores de Competitividade - and by national funds through FCT under the project PEst-C/EME/UI0285/2011.

[1] Di Xu, et al, Microelectronic Engineering, 85, 2155-2158, 2008.

[2] Xi Zhang, et al, Journal of Nanoscience and Nanotechnology, 8, 2568-2574, 2008.

[3] Guan-tai Lui, et al, Journal of Physics D: Applied Physics, 42, 215410, 2009.

[4] X. Zhang and A. Misra, Scripta Materialia, 66, 860-865, 2012. 

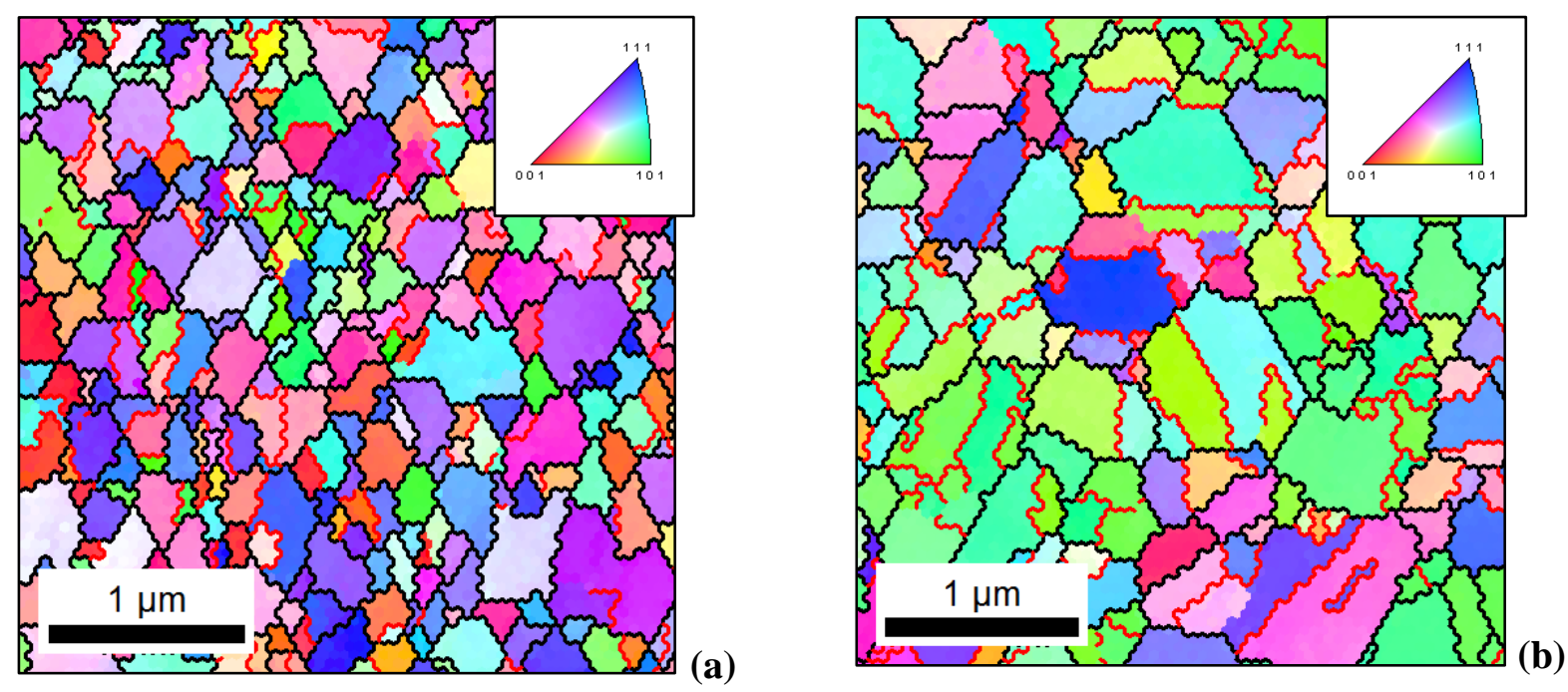

Figure 1. Orientation maps showing a finer microstructure for direct (a) than for pulsed (b) current films.

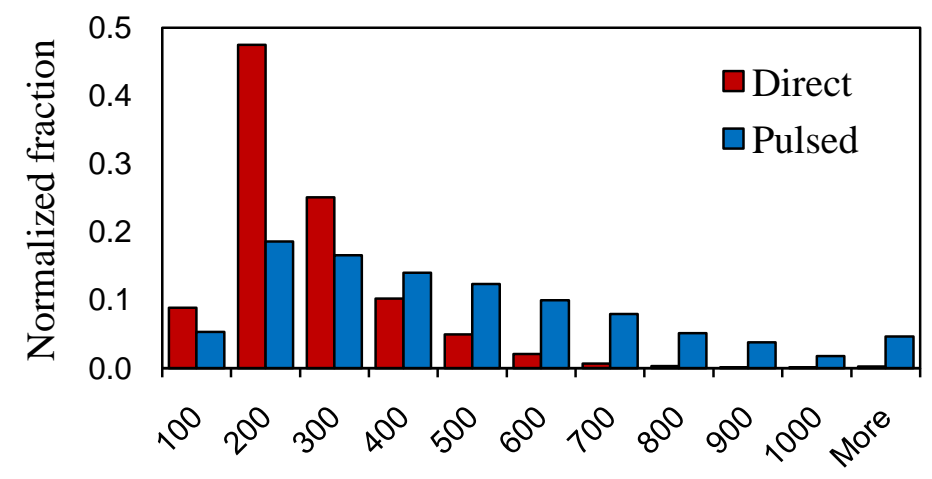

Grain size (nm)

Figure 2. Grain size distribution for direct and pulsed current.

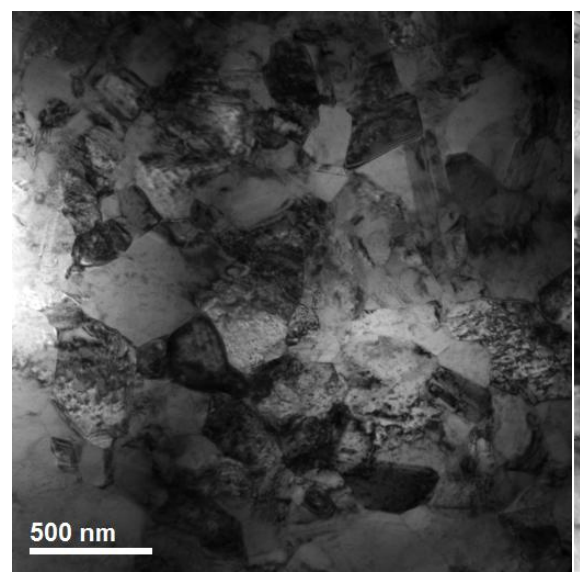

(a)

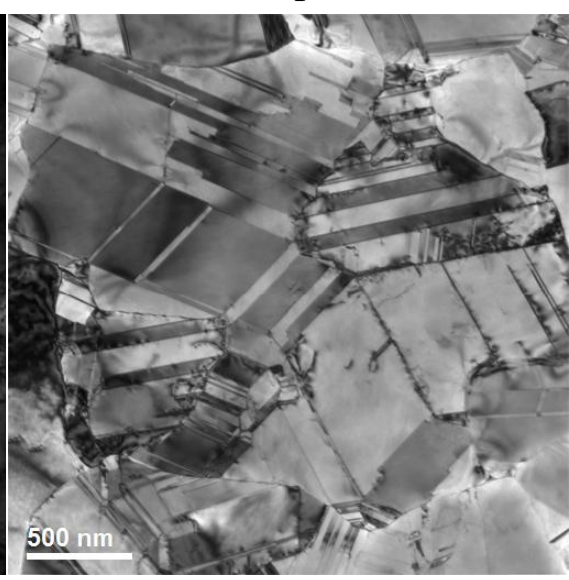

(b)

Figure 3. TEM images of (a) DC film showing a near twin-absent ultrafine grains microstructure while (b) PC film presents coarser grains containing many nanotwins. 